DOI 10.7603/s40956-016-0002-4

Moroccan J. Pure and Appl. Anal.(MJPAA)

RESEARCH ARTICLE

Volume 2(1), 2016, Pages 12-32

ISSN: $2351-8227$

\title{
Generalization of Some Inequalities for Differentiable Co-ordinated Convex Functions With Applications
}

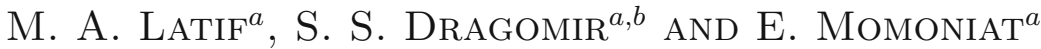

\begin{abstract}
In this paper, a new weighted identity for functions defined on a rectangle from the plane is established. By using the obtained identity and analysis, some new weighted integral inequalities for the classes of co-ordinated convex, co-ordinated wright-convex and co-ordinated quasi-convex functions on the rectangle from the plane are established which provide weighted generalization of some recent results proved for co-ordinated convex functions. Some applications of our results to random variables and $2 D$ weighted quadrature formula are given as well.

2000 Mathematics Subject Classification. 26D15, 26D20, $26 \mathrm{D} 07$.

Key words and phrases. Hermite-Hadamard's inequality, co-ordinated convex function, coordinated wright-convex function, co-ordinated quasi-convex function, Hölder's integral inequality, quadrature formula.
\end{abstract}

\section{Introduction}

The following definition is well known in mathematical analysis: A function $f: I \rightarrow \mathbb{R}, \emptyset \neq I \subseteq \mathbb{R}$, is said to be convex on $I$ if the inequality

$$
f(\lambda x+(1-\lambda) y) \leq \lambda f(x)+(1-\lambda) f(y),
$$

Received February 16, 2016 - Accepted March 30, 2016.

(C)The Author(s) 2016. This article is published with open access by Sidi Mohamed Ben Abdallah University

${ }^{a}$ School of Computational and Applied Mathematics, University of the Witwatersrand, Private Bag 3, Wits 2050, Johannesburg, South Africa.

e-mail: m_amer_latif@hotmail.com, ebrahim.momoniat@wits.ac.za.

${ }^{b}$ School of Engineering and Science, Victoria University, PO Box 14428 Melbourne City, MC 8001, Australia.

e-mail: sever.dragomir@vu.edu.au. 
holds for all $x, y \in I$ and $\lambda \in[0,1]$.

A number of results have been established for the class of convex functions but the most famous is the Hermite-Hadamard's inequality (see for instance [7]). This double inequality is stated as:

$$
f\left(\frac{a+b}{2}\right) \leq \frac{1}{b-a} \int_{a}^{b} f(x) d x \leq \frac{f(a)+f(b)}{2},
$$

where $f: I \rightarrow \mathbb{R}, \emptyset \neq I \subseteq \mathbb{R}$ a convex function, $a, b \in I$ with $a<b$. The inequalities in (1) are reversed if $f$ is a concave function.

The inequalities (1) have become an important cornerstone in mathematical analysis and optimization and many uses of these inequalities have been discovered in a variety of settings. Moreover, many inequalities of special means can be obtained for a particular choice of the function $f$. Due to the rich geometrical significance of Hermite-Hadamard's inequality (1), there is growing literature providing its new proofs, extensions, refinements and generalizations, see for example [2, 4, 5, 6, 9, 21, 22] and the references therein.

Let us consider now a bidimensional interval $[a, b] \times[c, d]$ in $\mathbb{R}^{2}$ with $a<b$ and $c<d$. A mapping $f:[a, b] \times[c, d] \rightarrow \mathbb{R}$ is said to be convex on $[a, b] \times[c, d]$ if the inequality

$$
f(\lambda x+(1-\lambda) z, \lambda y+(1-\lambda) w) \leq \lambda f(x, y)+(1-\lambda) f(z, w)
$$

holds for all $(x, y),(z, w) \in[a, b] \times[c, d]$ and $\lambda \in[0,1]$.

A modification for convex functions on $[a, b] \times[c, d]$, which are also known as coordinated convex functions, was initiated by Dragomir [4, 6] as follows:

A function $f:[a, b] \times[c, d] \rightarrow \mathbb{R}$ is said to be convex on the co-ordinates on $[a, b] \times[c, d]$ if the partial mappings $f_{y}:[a, b] \rightarrow \mathbb{R}, f_{y}(u)=f(u, y)$ and $f_{x}:[c, d] \rightarrow \mathbb{R}, f_{x}(v)=$ $f(x, v)$ are convex where defined for all $x \in[a, b], y \in[c, d]$.

A formal definition for co-ordinated convex functions may be stated as follows:

Definition 1.1. [13] A function $f:[a, b] \times[c, d] \rightarrow \mathbb{R}$ is said to be convex on the co-ordinates on $[a, b] \times[c, d]$ if the inequality

$$
\begin{aligned}
& f(t x+(1-t) y, s z+(1-s) w) \\
& \leq t s f(x, z)+t(1-s) f(x, w)+s(1-t) f(y, z)+(1-t)(1-s) f(y, w)
\end{aligned}
$$

holds for all $(t, s) \in[0,1] \times[0,1]$ and $(x, z),(y, w) \in[a, b] \times[c, d]$.

It has been proved in [4] that every convex mapping $f:[a, b] \times[c, d] \rightarrow \mathbb{R}$ is convex on the co-ordinates. Furthermore, there exists co-ordinated convex function which is not convex, (see for example $[4,6]$ ).

The following Hermite-Hadamard type inequality for co-ordinated convex functions on the rectangle from the plane $\mathbb{R}^{2}$ was also proved in [4]:

Theorem 1.1. [4] Suppose that $f:[a, b] \times[c, d] \rightarrow \mathbb{R}$ is co-ordinated convex on $[a, b] \times$ $[c, d]$. Then one has the inequalities:

$$
f\left(\frac{a+b}{2}, \frac{c+d}{2}\right)
$$




$$
\begin{aligned}
\leq \frac{1}{2}\left[\frac{1}{b-a} \int_{a}^{b} f\left(x, \frac{c+d}{2}\right) d x+\frac{1}{d-c} \int_{c}^{d} f\left(\frac{a+b}{2}, y\right) d y\right] \\
\leq \frac{1}{(b-a)(d-c)} \int_{a}^{b} \int_{c}^{d} f(x, y) d y d x \\
\leq \frac{1}{4}\left[\frac{1}{b-a} \int_{a}^{b} f(x, c) d x+\frac{1}{b-a} \int_{a}^{b} f(x, d) d x\right. \\
\left.+\frac{1}{d-c} \int_{c}^{d} f(a, y) d y+\frac{1}{d-c} \int_{c}^{d} f(b, y) d y\right] \\
\leq \frac{f(a, c)+f(a, d)+f(b, c)+f(b, d)}{4} .
\end{aligned}
$$

The above inequalities are sharp.

Sarikaya et al. [23], proved the following Hermite-Hadamard type inequalities.

Theorem 1.2. [23] Let $f:[a, b] \times[c, d] \subset \mathbb{R}^{2} \rightarrow \mathbb{R}$ be a partial differentiable mapping on $[a, b] \times[c, d]$ in $\mathbb{R}^{2}$ with $a<b, c<d$. If $\left|\frac{\partial^{2} f}{\partial s \partial t}\right|$ is convex on the co-ordinates on $[a, b] \times[c, d]$, then one has the inequalities:

$$
\begin{aligned}
\mid \frac{1}{(b-a)(d-c)} \int_{a}^{b} \int_{c}^{d} f(x, y) d y d x+\frac{f(a, c)+f(a, d)+f(b, c)+f(b, d)}{4} & \frac{1}{2}\left[\frac{1}{b-a} \int_{a}^{b} f(x, c) d x+\frac{1}{b-a} \int_{a}^{b} f(x, d) d x\right. \\
& \left.+\frac{1}{d-c} \int_{c}^{d} f(a, y) d y+\frac{1}{d-c} \int_{c}^{d} f(b, y) d y\right] \mid \\
\leq \frac{(b-a)(d-c)}{16} & {\left[\left|\frac{\partial^{2} f}{\partial s \partial t}(a, c)\right|+\left|\frac{\partial^{2} f}{\partial s \partial t}(a, d)\right|+\left|\frac{\partial^{2} f}{\partial s \partial t}(b, c)\right|+\left|\frac{\partial^{2} f}{\partial s \partial t}(b, d)\right|\right] . }
\end{aligned}
$$

The next two results from [23] involve powers of the absolute value of $\frac{\partial^{2} f}{\partial s \partial t}$.

Theorem 1.3. [23] Let $f:[a, b] \times[c, d] \subset \mathbb{R}^{2} \rightarrow \mathbb{R}$ be a partial differentiable mapping on $[a, b] \times[c, d]$ in $\mathbb{R}^{2}$ with $a<b, c<d$. If $\left|\frac{\partial^{2} f}{\partial s \partial t}\right|^{q}, q \geq 1$, is convex on the co-ordinates on $[a, b] \times[c, d]$, then one has the inequalities:

$$
\begin{aligned}
\mid \frac{1}{(b-a)(d-c)} \int_{a}^{b} \int_{c}^{d} f(x, y) d y d x+\frac{f(a, c)+f(a, d)+f(b, c)+f(b, d)}{4} \\
-\frac{1}{2}\left[\frac{1}{b-a} \int_{a}^{b} f(x, c) d x+\frac{1}{b-a} \int_{a}^{b} f(x, d) d x\right. \\
\left.\quad+\frac{1}{d-c} \int_{c}^{d} f(a, y) d y+\frac{1}{d-c} \int_{c}^{d} f(b, y) d y\right] \mid
\end{aligned}
$$




$$
\leq \frac{(b-a)(d-c)}{4(p+1)^{\frac{2}{p}}}\left[\frac{\left|\frac{\partial^{2} f}{\partial s \partial t}(a, c)\right|^{q}+\left|\frac{\partial^{2} f}{\partial s \partial t}(a, d)\right|^{q}+\left.\left|\frac{\partial^{2} f}{\partial s \partial t}(b, c)\right|\right|^{q}+\left|\frac{\partial^{2} f}{\partial s \partial t}(b, d)\right|^{q}}{4}\right]^{\frac{1}{q}}
$$

where $\frac{1}{p}+\frac{1}{q}=1$.

Theorem 1.4. [23] Let $f:[a, b] \times[c, d] \subset \mathbb{R}^{2} \rightarrow \mathbb{R}$ be a partial differentiable mapping on $[a, b] \times[c, d]$ in $\mathbb{R}^{2}$ with $a<b, c<d$. If $\left|\frac{\partial^{2} f}{\partial s \partial t}\right|^{q}, q>1$, is convex on the co-ordinates on $[a, b] \times[c, d]$, then one has the inequalities:

$$
\begin{aligned}
\mid \frac{1}{(b-a)(d-c)} \int_{a}^{b} \int_{c}^{d} f(x, y) d y d x+\frac{f(a, c)+f(a, d)+f(b, c)+f(b, d)}{4} & -\frac{1}{2}\left[\frac{1}{b-a} \int_{a}^{b} f(x, c) d x+\frac{1}{b-a} \int_{a}^{b} f(x, d) d x\right. \\
& \left.+\frac{1}{d-c} \int_{c}^{d} f(a, y) d y+\frac{1}{d-c} \int_{c}^{d} f(b, y) d y\right] \mid \\
\leq \frac{(b-a)(d-c)}{16} & {\left[\frac{\left|\frac{\partial^{2} f}{\partial s \partial t}(a, c)\right|^{q}+\left|\frac{\partial^{2} f}{\partial s \partial t}(a, d)\right|^{q}+\left|\frac{\partial^{2} f}{\partial s \partial t}(b, c)\right|^{q}+\left|\frac{\partial^{2} f}{\partial s \partial t}(b, d)\right|^{q}}{4}\right]^{\frac{1}{q}} . }
\end{aligned}
$$

In a recent paper [22], M. E. Özdemir et al. give the notion of co-ordinated quasiconvex functions which generalize the notion of co-ordinated convex functions.

Definition 1.2. [20] A function $f:[a, b] \times[c, d] \subset \mathbb{R}^{2} \rightarrow \mathbb{R}$ is said to be quasi-convex on $[a, b] \times[c, d]$ if the inequality

$$
f(\lambda x+(1-\lambda) z, \lambda y+(1-\lambda) w) \leq \max \{f(x, y), f(z, w)\}
$$

holds for all $(x, y),(z, w) \in[a, b] \times[c, d]$ and $\lambda \in[0,1]$.

A function $f:[a, b] \times[c, d] \rightarrow \mathbb{R}$ is said to be quasi-convex on the co-ordinates on $[a, b] \times[c, d]$ if the partial mappings $f_{y}:[a, b] \rightarrow \mathbb{R}, f_{y}(u)=f(u, y)$ and $f_{x}:[c, d] \rightarrow$ $\mathbb{R}, f_{x}(v)=f(x, v)$ are quasi-convex where defined for all $x \in[a, b], y \in[c, d]$.

Another way of describing the definition of co-ordinated quasi-convex functions is given below.

Definition 1.3. [16] A function $f:[a, b] \times[c, d] \subset \mathbb{R}^{2} \rightarrow \mathbb{R}$ is said to be quasi-convex on the co-ordinates on $[a, b] \times[c, d]$ if

$$
f(t x+(1-t) z, s y+(1-s) w) \leq \max \{f(x, y), f(x, w), f(z, y), f(z, w)\}
$$

for all $(x, y),(z, w) \in[a, b] \times[c, d]$ and $(s, t) \in[0,1] \times[0,1]$.

The class of co-ordinated quasi-convex functions on $[a, b] \times[c, d]$ is denoted by $Q C([a, b] \times[c, d])$. It has also been proved in $[20]$ that every quasi-convex functions on 
$[a, b] \times[c, d]$ is quasi-convex on the co-ordinates on $[a, b] \times[c, d]$. The following example reveals that there exists quasi-convex function on the co-ordinates which is not quasi-convex.

Example 1.1. [16] The function $f:[-2,2]^{2} \rightarrow \mathbb{R}$, defined by $f(x, y)=\lfloor x\rfloor\lfloor y\rfloor$, where $\lfloor\cdot\rfloor$ is the floor function. This function is quasi-convex on the co-ordinates on $[-2,2]^{2}$ but is not quasi-convex on $[0,1]^{2}$.

For example, take $(x, y)=(-2,1),(z, w)=(1,-1)$ and $\lambda=\frac{1}{2}$, then

$$
f(\lambda x+(1-\lambda) z, \lambda y+(1-\lambda) w)=f\left(-\frac{1}{2}, 0\right)=0,
$$

on the other hand

$$
\max \{f(x, y), f(z, w)\}=\max \{f(-2,1), f(1,-1)\}=-1,
$$

which shows that $f(\lambda x+(1-\lambda) z, \lambda y+(1-\lambda) w)>\max \{f(x, y), f(z, w)\}$.

Another generalization of the notion of the co-ordinated convex functions is the concept of wright-convex functions which is given in the definition below.

Definition 1.4. [20] A function $f:[a, b] \times[c, d] \subset \mathbb{R}^{2} \rightarrow \mathbb{R}$ is said to be wright-convex on $[a, b] \times[c, d]$ if the inequality

$$
\begin{aligned}
& f(\lambda x+(1-\lambda) z, \lambda y+(1-\lambda) w)+f((1-\lambda) x+\lambda z,(1-\lambda) y+\lambda w) \\
& \leq \max \{f(x, z), f(y, w)\},
\end{aligned}
$$

holds for all $(x, z),(y, w) \in[a, b] \times[c, d]$ and $\lambda \in[0,1]$.

A function $f:[a, b] \times[c, d] \rightarrow \mathbb{R}$ is said to be wright-convex on the co-ordinates on $[a, b] \times[c, d]$ if the partial mappings $f_{y}:[a, b] \rightarrow \mathbb{R}, f_{y}(u)=f(u, y)$ and $f_{x}:[c, d] \rightarrow$ $\mathbb{R}, f_{x}(v)=f(x, v)$ are wright-convex where defined for all $x \in[a, b], y \in[c, d]$.

The above definition of wright-convex functions on the co-ordinates can be reformulated as follows.

Definition 1.5. [20] A function $f:[a, b] \times[c, d] \subset \mathbb{R}^{2} \rightarrow \mathbb{R}$ is said to be wright-convex on the co-ordinates on $[a, b] \times[c, d]$ if

$$
\begin{aligned}
& f(t x+(1-t) z, s y+(1-s) w)+f((1-t) x+t z,(1-s) y+s w) \\
& \leq f(x, y)+f(z, y)+f(x, w)+f(z, w)
\end{aligned}
$$

for all $(x, z),(y, w) \in[a, b] \times[c, d]$ and $(s, t) \in[0,1] \times[0,1]$.

The class of co-ordinated wright-convex functions on $[a, b] \times[c, d]$ is represented by $W([a, b] \times[c, d])$. It has also been proved in $[20]$ that every wright-convex functions on $[a, b] \times[c, d]$ is wright-convex on the co-ordinates on $[a, b] \times[c, d]$.

For more recent results on co-ordinated convex, co-ordinated quasi-convex, co-ordinated $m$-convex, co-ordinated $(\alpha, m)$-convex and co-ordinated $s$-convex functions on a rectangle $[a, b] \times[c, d]$ from the plane $\mathbb{R}^{2}$, we refer the readers to $[1,5,8],[10]$-[20].

In the present paper, we establish a new weighted identity for differentiable mappings defined on a rectangle $[a, b] \times[c, d]$ from the plane $\mathbb{R}^{2}$ and by using the obtained 
identity and analysis, some new weighted integral inequalities for differentiable coordinated convex, co-ordinated wright-convex and co-ordinated quasi convex functions are proved. The results proved in the paper provide a weighted generalization of the results given in Theorem 1.2, Theorem 1.3 and Theorem 1.4. Applications of our results to random variables and $2 D$ weighted quadrature formula are provided as well.

\section{Main Results}

We need the following lemma to prove our results. Moreover, the following notions will be used throughout in the section

$$
\begin{aligned}
& U_{1}(a, b, t)=U_{1}(t)=\frac{1-t}{2} a+\frac{1+t}{2} b, L_{1}(a, b, t)=L_{1}(t)=\frac{1+t}{2} a+\frac{1-t}{2} b, \\
& U_{2}(c, d, s)=U_{2}(s)=\frac{1-s}{2} c+\frac{1+s}{2} d, L_{2}(c, d, s)=L_{2}(s)=\frac{1+s}{2} c+\frac{1-s}{2} d, \\
& \Psi\left(a, b, c, d ;\left|f_{t s}\right|\right)=\frac{\left|f_{t s}(a, c)\right|+\left|f_{t s}(a, d)\right|+\left|f_{t s}(b, c)\right|+\left|f_{t s}(b, d)\right|}{4}, \\
& \lambda_{1}\left(b, d, \frac{a+b}{2}, \frac{c+d}{2} ;\left|f_{t s}\right|\right) \\
& =\max \left\{\left|f_{t s}(b, d)\right|,\left|f_{t s}\left(b, \frac{c+d}{2}\right)\right|,\left|f_{t s}\left(\frac{a+b}{2}, d\right)\right|,\left|f_{t s}\left(\frac{a+b}{2}, \frac{c+d}{2}\right)\right|\right\}, \\
& \lambda_{2}\left(a, d, \frac{a+b}{2}, \frac{c+d}{2} ;\left|f_{t s}\right|\right) \\
& =\max \left\{\left|f_{t s}(a, d)\right|,\left|f_{t s}\left(a, \frac{c+d}{2}\right)\right|,\left|f_{t s}\left(\frac{a+b}{2}, d\right)\right|,\left|f_{t s}\left(\frac{a+b}{2}, \frac{c+d}{2}\right)\right|\right\}, \\
& \lambda_{3}\left(b, c, \frac{a+b}{2}, \frac{c+d}{2} ;\left|f_{t s}\right|\right) \\
& =\max \left\{\left|f_{t s}(b, c)\right|,\left|f_{t s}\left(b, \frac{c+d}{2}\right)\right|,\left|f_{t s}\left(\frac{a+b}{2}, c\right)\right|,\left|f_{t s}\left(\frac{a+b}{2}, \frac{c+d}{2}\right)\right|\right\}
\end{aligned}
$$

and

$$
\begin{aligned}
& \lambda_{4}\left(a, c, \frac{a+b}{2}, \frac{c+d}{2} ;\left|f_{t s}\right|\right) \\
& =\max \left\{\left|f_{t s}(a, c)\right|,\left|f_{t s}\left(a, \frac{c+d}{2}\right)\right|,\left|f_{t s}\left(\frac{a+b}{2}, c\right)\right|,\left|f_{t s}\left(\frac{a+b}{2}, \frac{c+d}{2}\right)\right|\right\} .
\end{aligned}
$$

Lemma 2.1. Let $f: \Delta \subset \mathbb{R}^{2} \rightarrow \mathbb{R}$ be a twice partially differentiable mapping on $\Delta^{\circ}$ and $p:[a, b] \times[c, d] \rightarrow[0, \infty)$ be continuous and symmetric to $\frac{a+b}{2}$ and $\frac{c+d}{2}$ for $[a, b] \times[c, d] \subset \Delta^{\circ}$ with $a<b, c<d$. If $f_{t s} \in L([a, b] \times[c, d])$, then

$$
\Phi(a, b, c, d ; p, f)=\frac{f(a, c)+f(a, d)+f(b, c)+f(b, d)}{4} \int_{c}^{d} \int_{a}^{b} p(x, y) d x d y
$$




$$
\begin{gathered}
-\frac{1}{2} \int_{c}^{d} \int_{a}^{b}[f(x, c)+f(x, d)] p(x, y) d x d y \\
-\frac{1}{2} \int_{c}^{d} \int_{a}^{b}[f(a, y)+f(b, y)] p(x, y) d x d y+\int_{c}^{d} \int_{a}^{b} f(x, y) p(x, y) d x d y \\
=\frac{(b-a)(d-c)}{16} \int_{0}^{1} \int_{0}^{1}\left[\int_{L_{2}(s)}^{U_{2}(s)} \int_{L_{1}(t)}^{U_{1}(t)} p(x, y) d x d y\right]\left[f_{t s}\left(U_{1}(t), U_{2}(s)\right)\right. \\
\left.-f_{t s}\left(U_{1}(t), L_{2}(s)\right)-f_{t s}\left(L_{1}(t), U_{2}(s)\right)+f_{t s}\left(L_{1}(t), L_{2}(s)\right)\right] d s d t .
\end{gathered}
$$

Proof. Let

$$
\begin{aligned}
I= & \frac{(b-a)(d-c)}{16} \int_{0}^{1} \int_{0}^{1}\left[\int_{L_{2}(s)}^{U_{2}(s)} \int_{L_{1}(t)}^{U_{1}(t)} p(x, y) d x d y\right]\left[f_{t s}\left(U_{1}(t), U_{2}(s)\right)\right. \\
& \left.-f_{t s}\left(U_{1}(t), L_{2}(s)\right)-f_{t s}\left(L_{1}(t), U_{2}(s)\right)+f_{t s}\left(L_{1}(t), L_{2}(s)\right)\right] d s d t
\end{aligned}
$$

and

$$
\int_{L_{2}(s)}^{U_{2}(s)} \int_{L_{1}(t)}^{U_{1}(t)} p(x, y) d x d y=q(t, s) .
$$

then

$$
\begin{aligned}
I= & \frac{(b-a)(d-c)}{16} \int_{0}^{1} \int_{0}^{1} q(t, s)\left[f_{t s}\left(U_{1}(t), U_{2}(s)\right)-f_{t s}\left(U_{1}(t), L_{2}(s)\right)\right. \\
& \left.-f_{t s}\left(L_{1}(t), U_{2}(s)\right)+f_{t s}\left(L_{1}(t), L_{2}(s)\right)\right] d s d t .
\end{aligned}
$$

Now by integration by parts and by using the symmetry of $p(x, y)$ about $x=\frac{a+b}{2}$ and $y=\frac{c+d}{2}$, we have

$$
\begin{aligned}
\frac{(b-a)(d-c)}{16} & \int_{0}^{1} \int_{0}^{1} q(t, s) f_{t s}\left(U_{1}(t), U_{2}(s)\right) d s d t \\
= & \frac{(b-a)(d-c)}{16} \int_{0}^{1}\left[\int_{0}^{1} q(t, s) f_{t s}\left(U_{1}(t), U_{2}(s)\right) d s\right] d t \\
& =\frac{(b-a)(d-c)}{16} \int_{0}^{1}\left[\left.\frac{2}{d-c} q(t, s) f_{t}\left(U_{1}(t), U_{2}(s)\right)\right|_{0} ^{1}\right. \\
& =\frac{(b-a)}{8} \int_{0}^{1}\left[f_{t}\left(U_{1}(t), d\right)\left(\int_{c}^{d} \int_{L_{1}(t)}^{U_{1}(t)} p(x, y) d x d y\right)\right. \\
-( & -c) \int_{0}^{1}\left(\int_{L_{1}(t)}^{U_{1}(t)} p\left(x, U_{2}(s)\right) d x f_{t}\left(U_{t}(t), U_{2}(s)\right) d s\right] d t \\
= & \frac{(b-a)}{8} \int_{0}^{1} f_{t}\left(U_{1}(t), d\right)\left(\int_{c}^{d} \int_{L_{1}(t)}^{U_{1}(t)} p(x, y) d x d y\right) d t
\end{aligned}
$$




$$
\begin{aligned}
&-\frac{(b-a)}{4} \int_{\frac{c+d}{2}}^{d} \int_{0}^{1}\left(\int_{L_{1}(t)}^{U_{1}(t)} p(x, y) d x\right) f_{t}\left(U_{1}(t), y\right) d t d y \\
&=\frac{1}{4} f(b, d) \int_{c}^{d} \int_{a}^{b} p(x, y) d x d y-\frac{1}{2} \int_{c}^{d} \int_{\frac{a+b}{2}}^{b} p(x, y) f(x, d) d x d y \\
& \quad-\frac{1}{2} \int_{\frac{c+d}{2}}^{d} \int_{a}^{b} p(x, y) f(b, y) d x d y+\int_{\frac{c+d}{2}}^{d} \int_{\frac{a+b}{2}}^{b} p(x, y) f(x, y) d x d y .
\end{aligned}
$$

Similarly, we have

$$
\begin{array}{r}
-\frac{(b-a)(d-c)}{16} \int_{0}^{1} \int_{0}^{1} q(t, s) f_{t s}\left(U_{1}(t), L_{2}(s)\right) d s d t \\
=\frac{1}{4} f(b, c) \int_{c}^{d} \int_{a}^{b} p(x, y) d x d y-\frac{1}{2} \int_{c}^{d} \int_{\frac{a+b}{2}}^{b} p(x, y) f(x, c) d x d y \\
\quad-\frac{1}{2} \int_{c}^{\frac{c+d}{2}} \int_{a}^{b} p(x, y) f(b, y) d x d y+\int_{c}^{\frac{c+d}{2}} \int_{\frac{a+b}{2}}^{b} p(x, y) f(x, y) d x d y \\
-\frac{(b-a)(d-c)}{16} \int_{0}^{1} \int_{0}^{1} q(t, s) f_{t s}\left(L_{1}(t), U_{2}(s)\right) d s d t \\
=\frac{1}{4} f(a, d) \int_{c}^{d} \int_{a}^{b} p(x, y) d x d y-\frac{1}{2} \int_{c}^{d} \int_{a}^{\frac{a+b}{2}} p(x, y) f(x, d) d x d y \\
-\frac{1}{2} \int_{\frac{c+d}{2}}^{d} \int_{a}^{b} p(x, y) f(a, y) d x d y+\int_{\frac{c+d}{2}}^{d} \int_{a}^{\frac{a+b}{2}} p(x, y) f(x, y) d x d y
\end{array}
$$

and

$$
\begin{aligned}
& \frac{(b-a)(d-c)}{16} \int_{0}^{1} \int_{0}^{1} q(t, s) f_{t s}\left(L_{1}(t), L_{2}(s)\right) d s d t \\
& \quad=\frac{1}{4} f(a, c) \int_{c}^{d} \int_{a}^{b} p(x, y) d x d y-\frac{1}{2} \int_{c}^{d} \int_{a}^{\frac{a+b}{2}} p(x, y) f(x, c) d x d y \\
& \quad-\frac{1}{2} \int_{c}^{\frac{c+d}{2}} \int_{a}^{b} p(x, y) f(a, y) d x d y+\int_{c}^{\frac{c+d}{2}} \int_{a}^{\frac{a+b}{2}} p(x, y) f(x, y) d x d y .
\end{aligned}
$$

Adding (7)-(10), we get the desired result.

Remark 2.1. If we take $p(x, y)=\frac{1}{(b-a)(d-c)}$ for all $(x, y) \in[a, b] \times[c, d]$ in Lemma 2.1, we get Lemma 1 from [23, page 139].

Now by using lemma 2.1, we present the main results of this section.

Theorem 2.1. Let $f: \Delta \subset \mathbb{R}^{2} \rightarrow \mathbb{R}$ be a twice partially differentiable mapping on $\Delta^{\circ}$ and $p:[a, b] \times[c, d] \rightarrow[0, \infty)$ be continuous and symmetric to $\frac{a+b}{2}$ and $\frac{c+d}{2}$ for 
$[a, b] \times[c, d] \subset \Delta^{\circ}$ with $a<b, c<d$. If $f_{t s} \in L([a, b] \times[c, d])$ and $\left|f_{t s}\right|$ is convex on the co-ordinates on $[a, b] \times[c, d]$, then

$$
\begin{aligned}
& |\Phi(a, b, c, d ; p, f)| \\
& \quad \leq \frac{(b-a)(d-c)}{4} \Psi\left(a, b, c, d ;\left|f_{t s}\right|\right) \int_{0}^{1} \int_{0}^{1} \int_{L_{2}(s)}^{U_{2}(s)} \int_{L_{1}(t)}^{U_{1}(t)} p(x, y) d x d y d t d s .
\end{aligned}
$$

Proof. Taking absolute value on both sides of (6) and using the properties of absolute value, we have

$$
\begin{aligned}
& |\Phi(a, b, c, d ; p, f)| \\
& \leq \frac{(b-a)(d-c)}{16} \int_{0}^{1} \int_{0}^{1}\left[\int_{L_{2}(s)}^{U_{2}(s)} \int_{L_{1}(t)}^{U_{1}(t)} p(x, y) d x d y\right]\left[\left|f_{t s}\left(U_{1}(t), U_{2}(s)\right)\right|\right. \\
& \left.\quad+\left|f_{t s}\left(U_{1}(t), L_{2}(s)\right)\right|+\left|f_{t s}\left(L_{1}(t), U_{2}(s)\right)\right|+\left|f_{t s}\left(L_{1}(t), L_{2}(s)\right)\right|\right] d s d t .
\end{aligned}
$$

By the convexity of $\left|f_{t s}\right|$ on the co-ordinates on $[a, b] \times[c, d]$, we have

$$
\begin{aligned}
\mid f_{t s}\left(U_{1}(t),\right. & \left.U_{2}(s)\right) \mid \\
\leq & \left(\frac{1-t}{2}\right)\left(\frac{1-s}{2}\right)\left|f_{t s}(a, c)\right|+\left(\frac{1-t}{2}\right)\left(\frac{1+s}{2}\right)\left|f_{t s}(a, d)\right| \\
& \quad+\left(\frac{1+t}{2}\right)\left(\frac{1-s}{2}\right)\left|f_{t s}(b, c)\right|+\left(\frac{1+t}{2}\right)\left(\frac{1+s}{2}\right)\left|f_{t s}(b, d)\right|,
\end{aligned}
$$

$$
\begin{aligned}
\mid f_{t s}\left(U_{1}(t)\right. & \left., L_{2}(s)\right) \mid \\
\leq & \left(\frac{1-t}{2}\right)\left(\frac{1+s}{2}\right)\left|f_{t s}(a, c)\right|+\left(\frac{1-t}{2}\right)\left(\frac{1-s}{2}\right)\left|f_{t s}(a, d)\right| \\
& \quad+\left(\frac{1+t}{2}\right)\left(\frac{1+s}{2}\right)\left|f_{t s}(b, c)\right|+\left(\frac{1+t}{2}\right)\left(\frac{1-s}{2}\right)\left|f_{t s}(b, d)\right|
\end{aligned}
$$

$\left|f_{t s}\left(L_{1}(t), U_{2}(s)\right)\right|$

$$
\begin{aligned}
\leq & \left(\frac{1+t}{2}\right)\left(\frac{1-s}{2}\right)\left|f_{t s}(a, c)\right|+\left(\frac{1+t}{2}\right)\left(\frac{1+s}{2}\right)\left|f_{t s}(a, d)\right| \\
& +\left(\frac{1-t}{2}\right)\left(\frac{1-s}{2}\right)\left|f_{t s}(b, c)\right|+\left(\frac{1-t}{2}\right)\left(\frac{1+s}{2}\right)\left|f_{t s}(b, d)\right|,
\end{aligned}
$$

and

$$
\begin{aligned}
\mid f_{t s}\left(L_{1}(t),\right. & \left.L_{2}(s)\right) \mid \\
\leq & \left(\frac{1+t}{2}\right)\left(\frac{1+s}{2}\right)\left|f_{t s}(a, c)\right|+\left(\frac{1+t}{2}\right)\left(\frac{1-s}{2}\right)\left|f_{t s}(a, d)\right| \\
& \quad+\left(\frac{1-t}{2}\right)\left(\frac{1+s}{2}\right)\left|f_{t s}(b, c)\right|+\left(\frac{1-t}{2}\right)\left(\frac{1-s}{2}\right)\left|f_{t s}(b, d)\right| .
\end{aligned}
$$


Using (13)-(16) in (12), we get (11).

Remark 2.2. If we take $p(x, y)=\frac{1}{(b-a)(d-c)}$ for all $(x, y) \in[a, b] \times[c, d]$ in Theorem 2.1, we get Theorem 1.2 from [23].

A more general result is given in the following theorem.

Theorem 2.2. Let $f: \Delta \subset \mathbb{R}^{2} \rightarrow \mathbb{R}$ be a twice partially differentiable mapping on $\Delta^{\circ}$ and $p:[a, b] \times[c, d] \rightarrow[0, \infty)$ be continuous and symmetric to $\frac{a+b}{2}$ and $\frac{c+d}{2}$ for $[a, b] \times[c, d] \subset \Delta^{\circ}$ with $a<b, c<d$. If $f_{t s} \in L([a, b] \times[c, d])$ and $\left|f_{t s}\right|^{q}$ is convex on the co-ordinates on $[a, b] \times[c, d]$ for $q \geq 1$, then

$$
\begin{aligned}
& |\Phi(a, b, c, d ; p, f)| \\
& \quad \leq \frac{(b-a)(d-c)}{4}\left[\Psi\left(a, b, c, d ;\left|f_{t s}\right|^{q}\right)\right]^{\frac{1}{q}} \int_{0}^{1} \int_{0}^{1} \int_{L_{2}(s)}^{U_{2}(s)} \int_{L_{1}(t)}^{U_{1}(t)} p(x, y) d x d y d s d t .
\end{aligned}
$$

Proof. Taking absolute value on both sides of (6), by using the properties of absolute value and the Hölder inequality, we have

$$
\begin{aligned}
|\Phi(a, b, c, d ; p, f)| & \leq \frac{(b-a)(d-c)}{16}\left(\int_{0}^{1} \int_{0}^{1}\left[\int_{L_{2}(s)}^{U_{2}(s)} \int_{L_{1}(t)}^{U_{1}(t)} p(x, y) d x d y\right] d s d t\right)^{1-\frac{1}{q}} \\
\times & {\left[\left(\int_{0}^{1} \int_{0}^{1}\left[\int_{L_{2}(s)}^{U_{2}(s)} \int_{L_{1}(t)}^{U_{1}(t)} p(x, y) d x d y\right]\left|f_{t s}\left(U_{1}(t), U_{2}(s)\right)\right|^{q} d s d t\right)^{\frac{1}{q}}\right.} \\
+ & \left(\int_{0}^{1} \int_{0}^{1}\left[\int_{L_{2}(s)}^{U_{2}(s)} \int_{L_{1}(t)}^{U_{1}(t)} p(x, y) d x d y\right]\left|f_{t s}\left(U_{1}(t), L_{2}(s)\right)\right|^{q} d s d t\right)^{\frac{1}{q}} \\
+ & \left(\int_{0}^{1} \int_{0}^{1}\left[\int_{L_{2}(s)}^{U_{2}(s)} \int_{L_{1}(t)}^{U_{1}(t)} p(x, y) d x d y\right]\left|f_{t s}\left(L_{1}(t), U_{2}(s)\right)\right|^{q} d s d t\right)^{\frac{1}{q}} \\
+ & \left.\left(\int_{0}^{1} \int_{0}^{1}\left[\int_{L_{2}(s)}^{U_{2}(s)} \int_{L_{1}(t)}^{U_{1}(t)} p(x, y) d x d y\right]\left|f_{t s}\left(L_{1}(t), L_{2}(s)\right)\right|^{q} d s d t\right)^{\frac{1}{q}}\right] .
\end{aligned}
$$

By the power-mean inequality $\left(a_{1}^{r}+a_{2}^{r}+a_{3}^{r}+a_{4}^{r} \leq 4^{1-r}\left(a_{1}+a_{2}+a_{3}+a_{4}\right)^{r}\right.$ for $a_{1}, a_{2}, a_{3}$, $a_{4}>0$ and $\left.r<1\right)$ and using the convexity of $\left|f_{t s}\right|^{q}$ on the co-ordinates on $[a, b] \times[c, d]$ for $q \geq 1$, we have

$$
\begin{aligned}
\left(\int_{0}^{1} \int_{0}^{1}\right. & {\left.\left[\int_{L_{2}(s)}^{U_{2}(s)} \int_{L_{1}(t)}^{U_{1}(t)} p(x, y) d x d y\right]\left|f_{t s}\left(U_{1}(t), U_{2}(s)\right)\right|^{q} d s d t\right)^{\frac{1}{q}} } \\
+ & \left(\int_{0}^{1} \int_{0}^{1}\left[\int_{L_{2}(s)}^{U_{2}(s)} \int_{L_{1}(t)}^{U_{1}(t)} p(x, y) d x d y\right]\left|f_{t s}\left(U_{1}(t), L_{2}(s)\right)\right|^{q} d s d t\right)^{\frac{1}{q}}
\end{aligned}
$$




$$
\begin{gathered}
+\left(\int_{0}^{1} \int_{0}^{1}\left[\int_{L_{2}(s)}^{U_{2}(s)} \int_{L_{1}(t)}^{U_{1}(t)} p(x, y) d x d y\right]\left|f_{t s}\left(L_{1}(t), U_{2}(s)\right)\right|^{q} d s d t\right)^{\frac{1}{q}} \\
\left.+\left(\int_{0}^{1} \int_{0}^{1}\left[\int_{L_{2}(s)}^{U_{2}(s)} \int_{L_{1}(t)}^{U_{1}(t)} p(x, y) d x d y\right]\left|f_{t s}\left(L_{1}(t), L_{2}(s)\right)\right|^{q} d s d t\right)^{\frac{1}{q}}\right] \\
\leq 4^{1-\frac{1}{q}}\left[\left|f_{t s}(a, c)\right|+\left|f_{t s}(a, d)\right|+\left|f_{t s}(b, c)\right|+\left|f_{t s}(b, d)\right|\right]^{\frac{1}{q}} \\
\times\left(\int_{0}^{1} \int_{0}^{1} \int_{L_{2}(s)}^{U_{2}(s)} \int_{L_{1}(t)}^{U_{1}(t)} p(x, y) d x d y d s d t\right)^{\frac{1}{q}} .
\end{gathered}
$$

A usage of (19) in (18) yields the desired result.

Remark 2.3. If we take $p(x, y)=\frac{1}{(b-a)(d-c)}$ for all $(x, y) \in[a, b] \times[c, d]$ in Theorem 2.2, we get Theorem 1.4.

A different approach leads to the following result.

Theorem 2.3. Let $f: \Delta \subset \mathbb{R}^{2} \rightarrow \mathbb{R}$ be a twice differentiable mapping on $\Delta^{\circ}$ and $p$ : $[a, b] \times[c, d] \rightarrow[0, \infty)$ be continuous and symmetric to $\frac{a+b}{2}$ and $\frac{c+d}{2}$ for $[a, b] \times[c, d] \subset \Delta^{\circ}$ with $a<b, c<d$. If $f_{t s} \in L([a, b] \times[c, d])$ and $\left|f_{t s}\right|^{q}$ is convex on the co-ordinates on $[a, b] \times[c, d]$ for $q>1$, then

$$
\begin{aligned}
& |\Phi(a, b, c, d ; p, f)| \\
\leq & \frac{(b-a)(d-c)}{4}\left[\Psi\left(a, b, c, d ;\left|f_{t s}\right|^{q}\right)\right]^{\frac{1}{q}}\left(\int_{0}^{1} \int_{0}^{1}\left[\int_{L_{2}(s)}^{U_{2}(s)} \int_{L_{1}(t)}^{U_{1}(t)} p(x, y) d x d y\right]^{p} d s d t\right)^{\frac{1}{p}}
\end{aligned}
$$

where $\frac{1}{p}+\frac{1}{q}=1$.

Proof. From Lemma 2.1 and the Hölder inequality, we have

$$
\begin{aligned}
& |\Phi(a, b, c, d ; p, f)| \leq \frac{(b-a)(d-c)}{16}\left(\int_{0}^{1} \int_{0}^{1}\left[\int_{L_{2}(s)}^{U_{2}(s)} \int_{L_{1}(t)}^{U_{1}(t)} p(x, y) d x d y\right]^{p} d s d t\right)^{\frac{1}{p}} \\
& \times\left[\left(\int_{0}^{1} \int_{0}^{1}\left|f_{t s}\left(U_{1}(t), U_{2}(s)\right)\right|^{q} d s d t\right)^{\frac{1}{q}}+\left(\int_{0}^{1} \int_{0}^{1}\left|f_{t s}\left(U_{1}(t), L_{2}(s)\right)\right|^{q} d s d t\right)^{\frac{1}{q}}\right. \\
& \left.\quad+\left(\int_{0}^{1} \int_{0}^{1}\left|f_{t s}\left(L_{1}(t), U_{2}(s)\right)\right|^{q} d s d t\right)^{\frac{1}{q}}+\left(\int_{0}^{1} \int_{0}^{1}\left|f_{t s}\left(L_{1}(t), L_{2}(s)\right)\right|^{q} d s d t\right)^{\frac{1}{q}}\right] .
\end{aligned}
$$

By the power-mean inequality $\left(a_{1}^{r}+a_{2}^{r}+a_{3}^{r}+a_{4}^{r} \leq 4^{1-r}\left(a_{1}+a_{2}+a_{3}+a_{4}\right)^{r}\right.$ for $a_{1}, a_{2}, a_{3}$, $a_{4}>0$ and $\left.r<1\right)$ and using the convexity of $\left|f_{t s}\right|^{q}$ on the co-ordinates on $[a, b] \times[c, d]$ for $q>1$, we have 


$$
\begin{gathered}
\left(\int_{0}^{1} \int_{0}^{1}\left|f_{t s}\left(U_{1}(t), U_{2}(s)\right)\right|^{q} d s d t\right)^{\frac{1}{q}}+\left(\int_{0}^{1} \int_{0}^{1}\left|f_{t s}\left(U_{1}(t), L_{2}(s)\right)\right|^{q} d s d t\right)^{\frac{1}{q}} \\
+\left(\int_{0}^{1} \int_{0}^{1}\left|f_{t s}\left(L_{1}(t), U_{2}(s)\right)\right|^{q} d s d t\right)^{\frac{1}{q}}+\left(\int_{0}^{1} \int_{0}^{1}\left|f_{t s}\left(L_{1}(t), L_{2}(s)\right)\right|^{q} d s d t\right)^{\frac{1}{q}} \\
\leq 4^{1-\frac{1}{q}}\left[\int_{0}^{1} \int_{0}^{1}\left|f_{t s}\left(U_{1}(t), U_{2}(s)\right)\right|^{q} d s d t+\int_{0}^{1} \int_{0}^{1}\left|f_{t s}\left(U_{1}(t), L_{2}(s)\right)\right|^{q} d s d t\right. \\
\left.+\int_{0}^{1} \int_{0}^{1}\left|f_{t s}\left(L_{1}(t), U_{2}(s)\right)\right|^{q} d s d t+\int_{0}^{1} \int_{0}^{1}\left|f_{t s}\left(L_{1}(t), L_{2}(s)\right)\right|^{q} d s d t\right]^{\frac{1}{q}} \\
\leq 4\left[\frac{\left|f_{t s}(a, c)\right|^{q}+\left|f_{t s}(a, d)\right|^{q}+\left|f_{t s}(b, c)\right|^{q}+\left|f_{t s}(b, d)\right|^{q}}{4}\right]^{\frac{1}{q}} .
\end{gathered}
$$

From (21) and (22), we get (20).

Remark 2.4. If we take $p(x, y)=\frac{1}{(b-a)(d-c)}$ for all $(x, y) \in[a, b] \times[c, d]$ in Theorem 2.3, we get Theorem 1.3.

Remark 2.5. Theorem 2.1-Theorem 2.3 continue to hold true if in their statements we replace the condition "convex on the co-ordinates" with the condition "wright-convex on the co-ordinates". However, the details are left to the interested reader.

In what follows we give our results for the quasi-convex mappings on the co-ordinates on $[a, b] \times[c, d]$.

Theorem 2.4. Suppose the assumptions of Theorem 2.1 are satisfied. If the mapping $\left|f_{t s}\right|$ is quasi-convex on the co-ordinates on $[a, b] \times[c, d]$, then the following inequality holds

$$
\begin{aligned}
& |\Phi(a, b, c, d ; p, f)| \leq \frac{(b-a)(d-c)}{16}\left[\lambda_{1}\left(b, d, \frac{a+b}{2}, \frac{c+d}{2} ;\left|f_{t s}\right|\right)\right. \\
& +\lambda_{2}\left(a, d, \frac{a+b}{2}, \frac{c+d}{2} ;\left|f_{t s}\right|\right)+\lambda_{3}\left(b, c, \frac{a+b}{2}, \frac{c+d}{2} ;\left|f_{t s}\right|\right) \\
& \left.+\lambda_{4}\left(a, c, \frac{a+b}{2}, \frac{c+d}{2} ;\left|f_{t s}\right|\right)\right] \int_{0}^{1} \int_{0}^{1} \int_{L_{2}(s)}^{U_{2}(s)} \int_{L_{1}(t)}^{U_{1}(t)} p(x, y) d x d y d t d s .
\end{aligned}
$$

Proof. We continue inequality (12) in the proof of Theorem 2.1. Now, by the quasiconvexity on the co-ordinates of $\left|f_{t s}\right|$ on $[a, b] \times[c, d]$, we obtain

$$
\begin{aligned}
& \left|f_{t s}\left(U_{1}(t), U_{2}(s)\right)\right| \\
& \leq \max \left\{\left|f_{t s}(b, d)\right|,\left|f_{t s}\left(b, \frac{c+d}{2}\right)\right|,\left|f_{t s}\left(\frac{a+b}{2}, d\right)\right|,\left|f_{t s}\left(\frac{a+b}{2}, \frac{c+d}{2}\right)\right|\right\}, \\
& \left|f_{t s}\left(L_{1}(t), U_{2}(s)\right)\right| \\
& \leq \max \left\{\left|f_{t s}(a, d)\right|,\left|f_{t s}\left(a, \frac{c+d}{2}\right)\right|,\left|f_{t s}\left(\frac{a+b}{2}, d\right)\right|,\left|f_{t s}\left(\frac{a+b}{2}, \frac{c+d}{2}\right)\right|\right\},
\end{aligned}
$$




$$
\begin{aligned}
& \left|f_{t s}\left(U_{1}(t), L_{2}(s)\right)\right| \\
& \quad \leq \max \left\{\left|f_{t s}(b, c)\right|,\left|f_{t s}\left(b, \frac{c+d}{2}\right)\right|,\left|f_{t s}\left(\frac{a+b}{2}, c\right)\right|,\left|f_{t s}\left(\frac{a+b}{2}, \frac{c+d}{2}\right)\right|\right\},
\end{aligned}
$$

and

$$
\begin{aligned}
& \left|f_{t s}\left(L_{1}(t), L_{2}(s)\right)\right| \\
& \quad \leq \max \left\{\left|f_{t s}(a, c)\right|,\left|f_{t s}\left(a, \frac{c+d}{2}\right)\right|,\left|f_{t s}\left(\frac{a+b}{2}, c\right)\right|,\left|f_{t s}\left(\frac{a+b}{2}, \frac{c+d}{2}\right)\right|\right\},
\end{aligned}
$$

for all $(t, s) \in[0,1] \times[0,1]$. A combination of (24)-(27) and (12)gives the required inequality (23).

Corollary 2.1. Suppose the assumptions of Theorem 2.4 are fulfilled and if $p(x, y)=$ $\frac{1}{(b-a)(d-c)}$ for all $(x, y) \in[a, b] \times[c, d]$, then the following inequality holds valid

$$
\begin{aligned}
& \mid \frac{f(a, c)+f(a, d)+f(b, c)+f(b, d)}{4} \\
& -\frac{1}{2(b-a)} \int_{a}^{b}[f(x, c)+f(x, d)] d x-\frac{1}{2(d-c)} \int_{c}^{d}[f(a, y)+f(b, y)] d y \\
& +\frac{1}{(b-a)(d-c)} \int_{c}^{d} \int_{a}^{b} f(x, y) d x d y \mid \leq \frac{(b-a)(d-c)}{16} \\
& \times\left[\lambda_{1}\left(b, d, \frac{a+b}{2}, \frac{c+d}{2} ;\left|f_{t s}\right|\right)+\lambda_{2}\left(a, d, \frac{a+b}{2}, \frac{c+d}{2} ;\left|f_{t s}\right|\right)\right. \\
& \left.+\lambda_{3}\left(b, c, \frac{a+b}{2}, \frac{c+d}{2} ;\left|f_{t s}\right|\right)+\lambda_{4}\left(a, c, \frac{a+b}{2}, \frac{c+d}{2} ;\left|f_{t s}\right|\right)\right] .
\end{aligned}
$$

Corollary 2.2. Suppose the assumptions of Theorem 2.4 are satisfied and additionally

(1) If $\left|f_{t s}\right|$ is non-decreasing on the co-ordinates on $[a, b] \times[c, d]$, then the following inequality holds true

$$
\begin{aligned}
& |\Phi(a, b, c, d ; p, f)| \\
& \leq \frac{(b-a)(d-c)}{16}\left[\left|f_{t s}(b, d)\right|+\left|f_{t s}\left(\frac{a+b}{2}, d\right)\right|+\left|f_{t s}\left(b, \frac{c+d}{2}\right)\right|\right. \\
& \left.+\left|f_{t s}\left(\frac{a+b}{2}, \frac{c+d}{2}\right)\right|\right] \int_{0}^{1} \int_{0}^{1} \int_{L_{2}(s)}^{U_{2}(s)} \int_{L_{1}(t)}^{U_{1}(t)} p(x, y) d x d y d t d s .
\end{aligned}
$$

(2) If $\left|f_{t s}\right|$ is non-increasing on the co-ordinates on $[a, b] \times[c, d]$, then the following inequality holds true

$$
\begin{aligned}
& |\Phi(a, b, c, d ; p, f)| \\
& \quad \leq \frac{(b-a)(d-c)}{16}\left[\left|f_{t s}(a, c)\right|+\left|f_{t s}\left(a, \frac{c+d}{2}\right)\right|+\left|f_{t s}\left(\frac{a+b}{2}, c\right)\right|\right.
\end{aligned}
$$




$$
\left.+\left|f_{t s}\left(\frac{a+b}{2}, \frac{c+d}{2}\right)\right|\right] \int_{0}^{1} \int_{0}^{1} \int_{L_{2}(s)}^{U_{2}(s)} \int_{L_{1}(t)}^{U_{1}(t)} p(x, y) d x d y d t d s .
$$

Corollary 2.3. If we take $p(x, y)=\frac{1}{(b-a)(d-c)}$ for all $(x, y) \in[a, b] \times[c, d]$ in Corollary 2.2 and additionally

(1) If $\left|f_{t s}\right|$ is non-decreasing on the co-ordinates on $[a, b] \times[c, d]$, then the following inequality holds true

$$
\begin{aligned}
& \mid \frac{f(a, c)+f(a, d)+f(b, c)+f(b, d)}{4} \\
& \quad-\frac{1}{2(b-a)} \int_{a}^{b}[f(x, c)+f(x, d)] d x-\frac{1}{2(d-c)} \int_{c}^{d}[f(a, y)+f(b, y)] d y \\
& \quad+\frac{1}{(b-a)(d-c)} \int_{c}^{d} \int_{a}^{b} f(x, y) d x d y \mid \leq \frac{(b-a)(d-c)}{16} \\
& \times\left[\left|f_{t s}(b, d)\right|+\left|f_{t s}\left(\frac{a+b}{2}, d\right)\right|+\left|f_{t s}\left(b, \frac{c+d}{2}\right)\right|+\left|f_{t s}\left(\frac{a+b}{2}, \frac{c+d}{2}\right)\right|\right] .
\end{aligned}
$$

(2) If $\left|f_{\text {ts }}\right|$ is non-increasing on the co-ordinates on $[a, b] \times[c, d]$, then the following inequality holds true

$$
\begin{aligned}
& \mid \frac{f(a, c)+f(a, d)+f(b, c)+f(b, d)}{4} \\
& \quad-\frac{1}{2(b-a)} \int_{a}^{b}[f(x, c)+f(x, d)] d x-\frac{1}{2(d-c)} \int_{c}^{d}[f(a, y)+f(b, y)] d y \\
& \quad+\frac{1}{(b-a)(d-c)} \int_{c}^{d} \int_{a}^{b} f(x, y) d x d y \mid \leq \frac{(b-a)(d-c)}{16} \\
& \times\left[\left|f_{t s}(a, c)\right|+\left|f_{t s}\left(a, \frac{c+d}{2}\right)\right|+\left|f_{t s}\left(\frac{a+b}{2}, c\right)\right|+\left|f_{t s}\left(\frac{a+b}{2}, \frac{c+d}{2}\right)\right|\right] .
\end{aligned}
$$

Theorem 2.5. Suppose the assumptions of Theorem 2.1 are satisfied. If the mapping $\left|f_{t s}\right|^{q}$ is quasi-convex on the co-ordinates on $[a, b] \times[c, d]$ for $q \geq 1$, then the following inequality holds

$$
\begin{aligned}
& |\Phi(a, b, c, d ; p, f)| \leq \frac{(b-a)(d-c)}{16}\left\{\left[\lambda_{1}\left(b, d, \frac{a+b}{2}, \frac{c+d}{2} ;\left|f_{t s}\right|^{q}\right)\right]^{\frac{1}{q}}\right. \\
& +\left[\lambda_{2}\left(a, d, \frac{a+b}{2}, \frac{c+d}{2} ;\left|f_{t s}\right|^{q}\right)\right]^{\frac{1}{q}}+\left[\lambda_{3}\left(b, c, \frac{a+b}{2}, \frac{c+d}{2} ;\left|f_{t s}\right|^{q}\right)\right]^{\frac{1}{q}} \\
& \left.+\left[\lambda_{4}\left(a, c, \frac{a+b}{2}, \frac{c+d}{2} ;\left|f_{t s}\right|^{q}\right)\right]^{\frac{1}{q}}\right] \int_{0}^{1} \int_{0}^{1} \int_{L_{2}(s)}^{U_{2}(s)} \int_{L_{1}(t)}^{U_{1}(t)} p(x, y) d x d y d t d s .
\end{aligned}
$$

Proof. We continue inequality (18) in the proof of Theorem 2.2. Now, by the quasiconvexity on the co-ordinates of $\left|f_{t s}\right|^{q}$ on $[a, b] \times[c, d]$ for $q \geq 1$ and the power-mean 
inequality, we obtain

$$
\begin{aligned}
& \left|f_{t s}\left(U_{1}(t), U_{2}(s)\right)\right|^{q} \\
& \quad \leq \max \left\{\left|f_{t s}(b, d)\right|^{q},\left|f_{t s}\left(b, \frac{c+d}{2}\right)\right|^{q},\left|f_{t s}\left(\frac{a+b}{2}, d\right)\right|^{q},\left|f_{t s}\left(\frac{a+b}{2}, \frac{c+d}{2}\right)\right|^{q}\right\}, \\
& \left|f_{t s}\left(L_{1}(t), U_{2}(s)\right)\right|^{q} \\
& \leq \max \left\{\left|f_{t s}(a, d)\right|^{q},\left|f_{t s}\left(a, \frac{c+d}{2}\right)\right|^{q},\left|f_{t s}\left(\frac{a+b}{2}, d\right)\right|^{q},\left|f_{t s}\left(\frac{a+b}{2}, \frac{c+d}{2}\right)\right|^{q}\right\}, \\
& \left|f_{t s}\left(U_{1}(t), L_{2}(s)\right)\right|^{q}, \\
& \quad \leq \max \left\{\left|f_{t s}(b, c)\right|^{q},\left|f_{t s}\left(b, \frac{c+d}{2}\right)\right|^{q},\left|f_{t s}\left(\frac{a+b}{2}, c\right)\right|^{q},\left|f_{t s}\left(\frac{a+b}{2}, \frac{c+d}{2}\right)\right|^{q}\right\},
\end{aligned}
$$

and

$$
\begin{aligned}
& \left|f_{t s}\left(L_{1}(t), L_{2}(s)\right)\right|^{q} \\
& \quad \leq \max \left\{\left|f_{t s}(a, c)\right|^{q},\left|f_{t s}\left(a, \frac{c+d}{2}\right)\right|^{q},\left|f_{t s}\left(\frac{a+b}{2}, c\right)\right|^{q},\left|f_{t s}\left(\frac{a+b}{2}, \frac{c+d}{2}\right)\right|^{q}\right\},
\end{aligned}
$$

for all $(t, s) \in[0,1] \times[0,1]$. Using (34)-(37) in (18) we get the desired result.

Corollary 2.4. Suppose the assumptions of Theorem 2.5 are fulfilled and if $p(x, y)=$ $\frac{1}{(b-a)(d-c)}$ for all $(x, y) \in[a, b] \times[c, d]$, then the following inequality holds valid

$$
\begin{aligned}
& \mid \frac{f(a, c)+f(a, d)+f(b, c)+f(b, d)}{4} \\
& \quad-\frac{1}{2} \int_{a}^{b}[f(x, c)+f(x, d)] d x-\frac{1}{2} \int_{c}^{d}[f(a, y)+f(b, y)] d y \\
& \quad+\int_{c}^{d} \int_{a}^{b} f(x, y) d x d y \mid \leq \frac{(b-a)(d-c)}{16}\left\{\left[\lambda_{1}\left(b, d, \frac{a+b}{2}, \frac{c+d}{2} ;\left|f_{t s}\right|^{q}\right)\right]^{\frac{1}{q}}\right. \\
& \quad+\left[\lambda_{2}\left(a, d, \frac{a+b}{2}, \frac{c+d}{2} ;\left|f_{t s}\right|^{q}\right)\right]^{\frac{1}{q}}+\left[\lambda_{3}\left(b, c, \frac{a+b}{2}, \frac{c+d}{2} ;\left|f_{t s}\right|^{q}\right)\right]^{\frac{1}{q}} \\
& \left.+\left[\lambda_{4}\left(a, c, \frac{a+b}{2}, \frac{c+d}{2} ;\left|f_{t s}\right|^{q}\right)\right]^{\frac{1}{q}}\right] \int_{0}^{1} \int_{0}^{1} \int_{L_{2}(s)}^{U_{2}(s)} \int_{L_{1}(t)}^{U_{1}(t)} p(x, y) d x d y d t d s .
\end{aligned}
$$

Remark 2.6. Suppose the assumptions of Theorem 2.5 are satisfied and additionally 
(1) If $\left|f_{t s}\right|^{q}$ is non-decreasing on the co-ordinates on $[a, b] \times[c, d]$, then (29) holds valid.

(2) If $\left|f_{t s}\right|^{q}$ is non-increasing on the co-ordinates on $[a, b] \times[c, d]$, then (30) holds true.

Remark 2.7. In Corollary 2.4

(1) If $\left|f_{t s}\right|^{q}$ is non-decreasing on the co-ordinates on $[a, b] \times[c, d]$, then (31) holds valid.

(2) If $\left|f_{t s}\right|^{q}$ is non-increasing on the co-ordinates on $[a, b] \times[c, d]$, then (32) holds true.

\section{Applications to Random Variables}

Let $0<a<b, 0<c<d, \alpha, \beta \in \mathbb{R}$ and let $X$ and $Y$ be two independent continuous random variables having the bi-variate continuous probability density function $p$ : $[a, b] \times[c, d] \rightarrow[0, \infty)$ which is symmetric to $\frac{a+b}{2}$ and $\frac{c+d}{2}$ the $\alpha$-moment of $X$ and the $\beta$-moment of $Y$ about the origin are respectively defined as follows

$$
E_{\alpha}(X)=\int_{c}^{b} t^{\alpha} p_{1}(t) d t, E_{\beta}(Y)=\int_{c}^{b} s^{\beta} p_{2}(s) d s
$$

which are assumed to be finite, here $p_{1}:[a, b] \rightarrow[0, \infty)$ and $p_{2}:[c, d] \rightarrow[0, \infty)$ are the marginal probability density functions of $X$ and $Y$. Since $X$ and $Y$ are independent random variables, we have

$$
p(t, s)=p_{1}(t) p_{2}(s)
$$

for all $(t, s) \in[a, b] \times[c, d]$.

Now we give some applications of our results to random variables.

Theorem 3.1. The inequality

$$
\begin{aligned}
\mid\left(E_{\alpha}(X)-\frac{a^{\alpha}+b^{\alpha}}{2}\right) & \left(E_{\beta}(Y)-\frac{c^{\beta}+d^{\beta}}{2}\right) \mid \\
\leq & \frac{(b-a)(d-c)}{4} \alpha \beta\left(\frac{a^{\alpha-1}+b^{\alpha-1}}{2}\right)\left(\frac{c^{\beta-1}+d^{\beta-1}}{2}\right) .
\end{aligned}
$$

holds holds for $0<a<b, 0<c<d$ and $\alpha, \beta \geq 2$.

Proof. Let $f(t, s)=t^{\alpha} s^{\beta}$ on $[a, b] \times[c, d]$ for $\alpha, \beta \geq 2$, we observe that $\left|f_{t s}(t, s)\right|=$ $\alpha \beta t^{\alpha-1} s^{\beta-1}$ is convex on the co-ordinates on $[a, b] \times[c, d]$. Since

$$
\begin{gathered}
\left|f_{t s}(a, c)\right|+\left|f_{t s}(a, d)\right|+\left|f_{t s}(b, c)\right|+\left|f_{t s}(b, d)\right| \\
=\alpha \beta\left(a^{\alpha-1}+b^{\alpha-1}\right)\left(c^{\beta-1}+d^{\beta-1}\right) \\
\int_{L_{2}(s)}^{U_{2}(s)} \int_{L_{1}(t)}^{U_{1}(t)} p(x, y) d x d y \leq \int_{c}^{d} \int_{a}^{b} p(x, y) d x d y=1
\end{gathered}
$$


and hence

$$
\int_{0}^{1} \int_{0}^{1} \int_{L_{2}(s)}^{U_{2}(s)} \int_{L_{1}(t)}^{U_{1}(t)} p(x, y) d x d y d t d s \leq 1
$$

Also

$$
\begin{gathered}
\frac{f(a, c)+f(a, d)+f(b, c)+f(b, d)}{4} \int_{c}^{d} \int_{a}^{b} p(x, y) d x d y \\
=\frac{a^{\alpha} c^{\beta}+a^{\alpha} d^{\beta}+b^{\alpha} c^{\beta}+b^{\alpha} d^{\beta}}{4}=\frac{\left(a^{\alpha}+b^{\alpha}\right)\left(c^{\beta}+d^{\beta}\right)}{4}, \\
\frac{1}{2} \int_{c}^{d} \int_{a}^{b}[f(x, c)+f(x, d)] p(x, y) d x d y+\frac{1}{2} \int_{c}^{d} \int_{a}^{b}[f(a, y)+f(b, y)] p(x, y) d x d y \\
=\left(\frac{c^{\beta}+d^{\beta}}{2}\right) E_{\alpha}(X)+\left(\frac{a^{\alpha}+b^{\alpha}}{2}\right) E_{\beta}(Y)
\end{gathered}
$$

and

$$
\int_{c}^{d} \int_{a}^{b} f(x, y) p(x, y) d x d y=E_{\alpha}(X) E_{\beta}(Y) .
$$

The result follows immediately from the inequality (11).

Theorem 3.2. The inequality

$$
\begin{aligned}
\mid\left(E_{\alpha}(X)\right. & \left.-\frac{a^{\alpha}+b^{\alpha}}{2}\right)\left(E_{\beta}(Y)-\frac{c^{\beta}+d^{\beta}}{2}\right) \mid \\
& \leq \frac{(b-a)(d-c)}{16} \alpha \beta\left(b^{\alpha-1}+\left(\frac{a+b}{2}\right)^{\alpha-1}\right)\left(d^{\beta-1}+\left(\frac{c+d}{2}\right)^{\beta-1}\right) .
\end{aligned}
$$

holds holds for $0<a<b, 0<c<d$ and $\alpha, \beta \geq 1$.

Proof. Let $f(t, s)=t^{\alpha} s^{\beta}$ on $[a, b] \times[c, d]$ for $\alpha, \beta \geq 1$, we observe that $\left|f_{t s}(t, s)\right|=$ $\alpha \beta t^{\alpha-1} s^{\beta-1}$ is non-decreasing and quasi-convex on the co-ordinates on $[a, b] \times[c, d]$. The proof is similar to that of Theorem 3.1 by using the inequality (29) we obtain the required result.

Remark 3.1. For $\alpha=\beta=1$, we have from Theorem 3.2 that

$$
\left|\left(E(X)-\frac{a+b}{2}\right)\left(E(Y)-\frac{c+d}{2}\right)\right| \leq \frac{(b-a)(d-c)}{4},
$$

where $E_{1}(X)=E(X)$ and $E_{1}(Y)=E(Y)$ are the expectation of the random variables $X$ and $Y$ respectively. 


\section{Applications to $2 \mathrm{D}$ weighted trapezoidal formula}

Let $[a, b] \times[c, d]$ be a rectangle from the plane $\mathbb{R}^{2}$. Suppose $d_{1}$ and $d_{2}$ are the divisions $a=x_{0}<x_{1}<\cdots<x_{n-1}<x_{n}=b$ and $c=y_{0}<y_{1}<\cdots<y_{m-1}<y_{m}=b$ of the intervals $[a, b]$ and $[c, d]$ respectively and let $\Omega=\left\{\left[x_{i}, x_{i+1}\right] \times\left[y_{j}, y_{j+1}\right]: 0 \leq i \leq n-1,0 \leq j \leq m-1\right\}$ be a corresponding division of the rectangle $[a, b] \times[c, d]$ from the plane $\mathbb{R}^{2}$.

Consider the following $2 D$ weighted quadrature formula

$$
\int_{c}^{d} \int_{a}^{b} f(x, y) p(x, y) d x d y=T(f, p, \Omega)+E(f, p, \Omega),
$$

where

$$
\begin{array}{r}
T(f, p, \Omega) \\
=-\sum_{i=0}^{n-1} \sum_{j=0}^{m-1}\left[\frac{f\left(x_{i}, y_{j}\right)+f\left(x_{i}, y_{j+1}\right)+f\left(x_{i+1}, y_{j}\right)+f\left(x_{i+1}, y_{j+1}\right)}{4}\right. \\
\times \int_{y_{j}}^{y_{j+1}} \int_{x_{i}}^{x_{i+1}} p(x, y) d x d y+\frac{1}{2} \int_{y_{j}}^{y_{j+1}} \int_{x_{i}}^{x_{i+1}}\left[f\left(x, y_{j}\right)+f\left(x, y_{j+1}\right)\right] p(x, y) d x d y \\
\left.+\frac{1}{2} \int_{y_{j}}^{y_{j+1}} \int_{x_{i}}^{x_{i+1}}\left[f\left(x_{i}, y\right)+f\left(x_{i+1}, y\right)\right] p(x, y) d x d y\right]
\end{array}
$$

for the trapezoidal version and $E(f, p, \Omega)$ denotes the associated approximation error.

The following results provide some estimates of the remainder term $E(f, p, \Omega)$.

Theorem 4.1. Suppose the assumptions of Theorem 2.2 are satisfied. If $\left|f_{t s}\right|^{q}$ is convex on the co-ordinates on $[a, b] \times[c, d]$ for $q \geq 1$, then in (42), for every division $\Omega$ of the rectangle $[a, b] \times[c, d]$ from the plane $\mathbb{R}^{2}$, the following holds

$$
\begin{aligned}
& |E(f, p, \Omega)| \leq \frac{1}{4} \sum_{i=0}^{n-1} \sum_{j=0}^{m-1}\left(x_{i+1}-x_{i}\right)\left(y_{j+1}-y_{j}\right) \\
& \quad \times\left[\Psi\left(x_{i}, x_{i+1}, y_{j}, y_{j+1} ;\left|f_{t s}\right|^{q}\right)\right]^{\frac{1}{q}} \int_{0}^{1} \int_{0}^{1} \int_{L_{2}\left(y_{j}, y_{j+1}, s\right)}^{U_{2}\left(y_{j}, y_{j+1}, s\right)} \int_{L_{1}\left(x_{i}, x_{i+1}, t\right)}^{U_{1}\left(x_{i}, x_{i+1}, t\right)} p(x, y) d x d y d s d t .
\end{aligned}
$$

Proof. Applying Theorem 2.2 on the rectangles $\left[x_{i}, x_{i+1}\right] \times\left[y_{j}, y_{j+1}\right](0 \leq i \leq n-1,0 \leq$ $j \leq m-1)$ of the division $\Omega$ of the rectangle $[a, b] \times[c, d]$ from the plane $\mathbb{R}^{2}$, we get

$$
\begin{aligned}
&\left|\Phi\left(x_{i}, x_{i+1}, y_{j}, y_{j+1} ; p, f\right)\right| \leq \frac{\left(x_{i+1}-x_{i}\right)\left(y_{j+1}-y_{j}\right)}{4} \\
& \times\left[\frac{\left|f_{t s}\left(x_{i}, y_{j}\right)\right|^{q}+\left|f_{t s}\left(x_{i}, y_{j+1}\right)\right|^{q}+\left|f_{t s}\left(x_{i+1}, y_{j}\right)\right|^{q}+\left|f_{t s}\left(x_{i+1}, y_{j+1}\right)\right|^{q}}{4}\right]^{\frac{1}{q}} \\
& \times \int_{0}^{1} \int_{0}^{1} \int_{L_{2}\left(y_{j}, y_{j+1}, s\right)}^{U_{2}\left(y_{j}, y_{j+1}, s\right)} \int_{L_{1}\left(x_{i}, x_{i+1}, t\right)}^{U_{1}\left(x_{i}, x_{i+1}, t\right)} p(x, y) d x d y d s d t .
\end{aligned}
$$


Summing over $i$ from 0 to $n-1$ and $j$ over 0 to $m-1$, we deduce, by the triangle inequality, that (44) holds.

Remark 4.1. The inequality holds if the condition of convexity of $\left|f_{t s}\right|^{q}$ on the coordinates on $[a, b] \times[c, d]$ is replaced with the condition of wright-convexity of $\left|f_{t s}\right|^{q}$ on the co-ordinates on $[a, b] \times[c, d]$ for $q \geq 1$.

Theorem 4.2. Suppose the assumptions of Theorem 2.2 are satisfied. If $\left|f_{t s}\right|^{q}$ is convex on the co-ordinates on $[a, b] \times[c, d]$ for $q \geq 1$, then in (42), for every division $\Omega$ of the rectangle $[a, b] \times[c, d]$ from the plane $\mathbb{R}^{2}$, the following holds

$$
\begin{aligned}
|E(f, p, \Omega)| \leq \frac{1}{16} \sum_{i=0}^{n-1} \sum_{j=0}^{m-1}\left(x_{i+1}-x_{i}\right)\left(y_{j+1}-y_{j}\right) \\
\times\left\{\left[\lambda_{1}\left(x_{i+1}, y_{j+1}, \frac{x_{i}+x_{i+1}}{2}, \frac{y_{j}+y_{j+1}}{2} ;\left|f_{t s}\right|^{q}\right)\right]^{\frac{1}{q}}\right. \\
+\left[\lambda_{2}\left(x_{i}, y_{j+1}, \frac{x_{i}+x_{i+1}}{2}, \frac{y_{j}+y_{j+1}}{2} ;\left|f_{t s}\right|^{q}\right)\right]^{\frac{1}{q}} \\
+\left[\lambda_{3}\left(x_{i+1}, y_{j}, \frac{x_{i}+x_{i+1}}{2}, \frac{y_{j}+y_{j+1}}{2} ;\left|f_{t s}\right|^{q}\right)\right]^{\frac{1}{q}} \\
\left.+\left[\lambda_{4}\left(x_{i}, y_{j}, \frac{x_{i}+x_{i+1}}{2}, \frac{y_{j}+y_{j+1}}{2} ;\left|f_{t s}\right|^{q}\right)\right]^{\frac{1}{q}}\right] \\
\quad \times \int_{0}^{1} \int_{0}^{1} \int_{L_{2}\left(y_{j}, y_{j+1}, s\right)}^{U_{2}\left(y_{j}, y_{j+1}, s\right)} \int_{L_{1}\left(x_{i}, x_{i+1}, t\right)}^{U_{1}\left(x_{i}, x_{i+1}, t\right)} p(x, y) d x d y d s d t .
\end{aligned}
$$

Proof. The proof follows from (33) by using the similar arguments as that of the proof of Theorem 4.1.

Remark 4.2. If $\left|f_{t s}\right|$ is non-decreasing in Theorem 4.2, then the following inequality holds

$$
\begin{aligned}
& |E(f, p, \Omega)| \leq \frac{1}{16} \sum_{i=0}^{n-1} \sum_{j=0}^{m-1}\left(x_{i+1}-x_{i}\right)\left(y_{j+1}-y_{j}\right) \\
& \quad \times\left[\left|f_{t s}\left(x_{i+1}, y_{j+1}\right)\right|+\left|f_{t s}\left(\frac{x_{i}+x_{i+1}}{2}, y_{j+1}\right)\right|+\left|f_{t s}\left(x_{i+1}, \frac{y_{j}+y_{j+1}}{2}\right)\right|\right. \\
& \left.+\left|f_{t s}\left(\frac{x_{i}+x_{i+1}}{2}, \frac{y_{j}+y_{j+1}}{2}\right)\right|\right] \int_{0}^{1} \int_{0}^{1} \int_{L_{2}\left(y_{j}, y_{j+1}, s\right)}^{U_{2}\left(y_{j}, y_{j+1}, s\right)} \int_{L_{1}\left(x_{i}, x_{i+1}, t\right)}^{U_{1}\left(x_{i}, x_{i+1}, t\right)} p(x, y) d x d y d s d t .
\end{aligned}
$$

and if $\left|f_{t s}\right|$ is non-increasing in Theorem 4.2, then the following inequality holds 


$$
\begin{aligned}
&|E(f, p, \Omega)| \leq \frac{1}{16} \sum_{i=0}^{n-1} \sum_{j=0}^{m-1}\left(x_{i+1}-x_{i}\right)\left(y_{j+1}-y_{j}\right) \\
& \times\left[\left|f_{t s}\left(x_{i}, y_{j}\right)\right|+\left|f_{t s}\left(x_{i}, \frac{y_{j}+y_{j+1}}{2}\right)\right|+\left|f_{t s}\left(\frac{x_{i}+x_{i+1}}{2}, y_{j}\right)\right|\right. \\
&\left.+\left|f_{t s}\left(\frac{x_{i}+x_{i+1}}{2}, \frac{y_{j}+y_{j+1}}{2}\right)\right|\right] \int_{0}^{1} \int_{0}^{1} \int_{L_{2}\left(y_{j}, y_{j+1}, s\right)}^{U_{2}\left(y_{j}, y_{j+1}, s\right)} \int_{L_{1}\left(x_{i}, x_{i+1}, t\right)}^{U_{1}\left(x_{i}, x_{i+1}, t\right)} p(x, y) d x d y d s d t .
\end{aligned}
$$

\section{References}

[1] M. Alomari and M. Darus, Fejer inequality for double integrals, Facta Universitatis (NIŠ): Ser. Math. Inform. 24(2009), 15-28.

[2] M. Alomari, M. Darus, U.S. Kirmaci, Refinements of Hadamard-type inequalities for quasi-convex functions with applications to trapezoidal formula and to special means, Computers \& Mathematics with Applications, Volume 59, Issue 1, January 2010, Pages 225-232

[3] S. S. Dragomir and R. P. Agarwal, Two inequalities for differentiable mappings and applications to special means of real numbers and to Trapezoidal formula, Appl. Math. Lett. 11(5) (1998) 91-95.

[4] S.S. Dragomir, On Hadamard's inequality for convex functions on the co-ordinates in a rectangle from the plane, Taiwanese Journal of Mathematics, 4 (2001), 775-788.

[5] S. S. Dragomir, Two mappings in connection to Hadamard's inequalities, Journal of Mathematical Analysis and Applications, 167, 49-56. http://dx.doi.org/10.1016/0022-247X(92)90233-4

[6] S.S. Dragomir and C.E.M. Pearce, Selected Topics on Hermite-Hadamard Inequalities and Applications, RGMIA Monographs, Victoria University, 2000. Online: [http://www.staff.vu.edu.au/RGMIA/monographs/hermite_hadamard.html] .

[7] J. Hadamard, Étude sur les Propriétés des Fonctions Entières en Particulier d'une Fonction Considérée par Riemann. Journal de Mathématiques Pures et Appliquées, 58, 171-215.

[8] D. Y. Hwang, K. L. Tseng, and G. S. Yang, Some Hadamard's inequalities for co-ordinated convex functions in a rectangle from the plane, Taiwanese Journal of Mathematics, 11(2007), 63-73.

[9] D. Y. Hwang, Some inequalities for differentiable convex mapping with application to weighted trapezoidal formula and higher moments of random variables, Applied Mathematics and Computation 217 (2011) 9598-9605.

[10] D. -Y. Hwang, K.-C. Hsu and K.-L. Tseng, Hadamard-Type inequalities for Lipschitzian functions in one and two variables with applications, Journal of Mathematical Analysis and Applications, 405, 546-554. http://dx.doi.org/10.1016/j.jmaa.2013.04.032.

[11] K.-C. Hsu, Some Hermite-Hadamard type inequalities for differentiable co-ordinated convex functions and applications, Advances in Pure Mathematics, 2014, 4, 326-340.

[12] K.-C. Hsu, Refinements of Hermite-Hadamard type inequalities for differentiable coordinated convex functions and applications, Taiwanese Journal of Mathematics, (In press). http://dx.doi.org/10.1142/9261.

[13] M. A. Latif and M. Alomari, Hadamard-type inequalities for product of two convex functions on the co-ordinates, Int. Math. Forum, 4(47), 2009, 2327-2338.

[14] M. A. Latif and M. Alomari, On the Hadamard-type inequalities for $h$-convex functions on the co-ordinates, Int. J. of Math. Analysis, 3(33), 2009, 1645-1656.

[15] M. A. Latif, S. S. Dragomir, On some new inequalities for differentiable co-ordinated convex functions, Journal of Inequalities and Applications 2012, 2012:28. 
[16] M. A. Latif, S. Hussain and S. S. Dragomir, Refinements of Hermite-Hadamard type inequalities for co-ordinated quasi-convex functions, International Journal of Mathematical Archive-3(1), 2012, 161-171.

[17] S.-L. Lyu, On the Hermite-Hadamard inequality for convex functions of two variable, Numerical Algebra, Control and Optimization, Volume 4, Number 1, March 2014.

[18] M.E. Özdemir, E. Set and M.Z. Sarikaya, New some Hadamard's type inequalities for co-ordinated $m$-convex and $(\alpha, m)$-convex functions, Hacettepe Journal of Mathematics and Statistics 40 (2), 219-229.

[19] M.E. Özdemir, M. A. Latif and A. O. Akdemir, On some Hadamard-type inequalities for product of two s-convex functions on the co-ordinates, Journal of Inequalities and Applications 2012, 2012:21. doi:10.1186/1029-242X-2012-21.

[20] M.E. Özdemir, A. O. Akdemir, Ağrı, C. Yıldız and Erzurum, On co-ordinated quasi-convex functions, Czechoslovak Mathematical Journal, 62 (137) (2012), 889-900.

[21] C. M. E. Pearce and J. E. Pečarić, Inequalities for differentiable mappings with applications to special means and quadrature formula, Appl. Math. Lett. 13 (2000) 51-55.

[22] J. E. Pečarić, F. Proschan and Y. L. Tong, Convex Functions, Partial Ordering and Statistical Applications, Academic Press, New York, 1991.

[23] M.Z. Sarikaya, E. Set, M.E. Özdemir and S. S. Dragomir, New some Hadamard's type inequalities for co-ordinated convex functions, Tamsui Oxford Journal of Information and Mathematical Sciences 28(2) (2012) 137-152. 